\title{
Impact of a Nationwide Measles Immunization Campaign and Routine Immunization in Nigeria, 2006-2010: A Critical Review of South-South, Nigeria
}

\author{
Bassey Enya Bassey*, Alex Gasasira, Goitom Weldegbriel, Maleghemi Toritseju Sylvester, \\ Koko I. Richard, Igbu Thompson, Ayodele Benjamin, Sylvester Agwai, Godwin Ubong Akpan
}

World Health Organisation off Yakubu Gowon Crescent Asokoro, Abuja, Nigeria

\section{Email address:}

bassey69@yahoo.com (B. E. Bassey), gasasiraa@yahoo.co.uk (A. Gasasira), weldegebrielg@ng.afro.who.int (G. Weldegbriel), Maleghemis@ng.afro.who.int (M. T. Sylvester), kokoi@ng.afro.who.int (K. I. Richard), igbut@ng.afro.who.int (I. Thompson), ayodeleb@ng.afro.who.int (A. Benjamin), agwais@ng.afro.who.int (S. Agwai), gees_kreation@yahoo.com (G. U. Akpan)

\section{To cite this article:}

Bassey Enya Bassey, Alex Gasasira, Goitom Weldegbriel, Maleghemi Toritseju Sylvester, Koko I. Richard, Igbu Thompson, Ayodele Benjamin, Sylvester Agwai, Godwin Ubong Akpan. Impact of a Nationwide Measles Immunization Campaign and Routine Immunization in Nigeria, 2006-2010: A Critical Review of South-South, Nigeria. Science Journal of Public Health. Vol. 3, No. 5, 2015 , pp. 693-698. doi: $10.11648 /$ j.sjph.20150305.25

\begin{abstract}
Background: Measles remains a serious problem of infancy and childhood in the developing world, despite the availability of vaccine. Increasing urbanization is changing patterns of endemicity. Objective: This paper critically examines the epidemiological impact of this nationwide measles immunization campaign and routine immunization, while taking into account any changes in surveillance performance. Methods: Blood samples were obtained from 4159 client at the surveillance focal sites scattered across the 123 district (LGAs) and were tested for measles specific immunoglobulin M (IgM). Five (5) ml of blood was collected from each subject into plain sterile bottle following informed consent. Blood samples were centrifuged and sera were separated and stored at $-20^{\circ} \mathrm{C}$ until used. Samples were analyzed in batches for measles specific IgM using commercial ELISA (MV-ELISA) (Enzygnost; Behring Diagnostics, Marburg, Germany) in accordance with the manufacturer's instructions. Tests were read on a pre-programmed spectrophotometer Quantum II, wavelength 450/630nm, manufactured by Abbott. Results: In total, 465 (11.2\%) tested positive for measles specific IgM antibodies. Of these, 1962 were male and 2197 were female. The highest number of IgM positive cases was found in those less than 5 years $(79.8 \%)$, while those aged 5-15 years, and 15 years and above recorded $17.2 \%$ and $3.0 \%$ respectively. The distribution of measles burden between urban and rural setting indicates that urban dwellers 53.8\% were more susceptible to measles than rural dwellers (46.2\%), this relationship was established as statistically significant with $(\mathrm{p}<0.0001)$ and odds ratio was also high 1.669 (95\% CI 1.375 2.025). A high significance of association between development of measles and vaccination status of subjects is also observed in this study $(\mathrm{p}<0.0001)$, while odds ratio was also observed to be high 6.144 (95\% CI 4.977-7.511). Conclusions: Improved understanding of measles epidemiology and risk factors are prerequisites for effective control. Possible strategies should include vertical vaccination efforts in addition to routine programmes.
\end{abstract}

Keywords: Measles, Immunization, Seasonal Variation

\section{Introduction}

Measles has been a notifiable disease in Nigeria since 1988 after the 1987 Yellow Fever outbreak [1]. In 2006 Nigeria implemented a measles case-based surveillance programme, coordinated by the World Health Organization (WHO). The WHO standard case definitions have been adopted (a suspected measles case was defined in one of two ways: (a) any person with fever and a maculopapular (i.e. non-vesicular) rash accompanied by cough, coryza or conjunctivitis; (b) any person suspected by a clinician of having measles.) [2]. Measles case-based surveillance requires monitoring consecutive stages of the elimination by tracking secondary outbreak cases, and serological 
testing of all suspected cases of measles [2]. Measles case based notification uses the following core indicators; the proportion of suspected measles cases investigated with blood specimens collected and transmitted to the laboratory for confirmation, the proportion of Local Government Areas (districts) that have investigated at least one suspected case of measles with a blood specimen within a year and the non-measles febrile rash illness rate per 100 , 000 population [2]. Following the introduction of routine immunisation for children under 1 year old, the accelerated measles campaign in the nineteen northern states in December 2005 and seventeen southern states in October 2006 which targeted those aged 9 months to 15 years, the incidence of measles decreased below the elimination threshold of one case per million inhabitants [3]. However, since 2008 the measles incidence has increased and remained above this elimination indicator [3].

This paper critically examines the epidemiological impact of this nationwide measles immunization campaign and routine immunization, while taking into account any changes in surveillance performance.

\section{Methods}

\subsection{Sample Collection and Analysis}

Five (5) mls of blood were collected from each subject into plain sterile bottle following informed consent. Blood samples were centrifuged and sera were separated and stored at $-20 \mathrm{oC}$ until used. Samples were analyzed in batches for measles specific IgM using commercial ELISA (MV-ELISA) (Enzygnost; Behring Diagnostics, Marburg, Germany) in accordance with the manufacturer's instructions. Tests were read on a pre-programmed spectrophotometer Quantum II, wavelength 450/630nm, manufactured by Abbott. We also analysed the 2006-2010 measles surveillance data from the zone. In assessing the campaign's epidemiological impact, only measles cases confirmed by laboratory results were included. A descriptive epidemiological analysis of confirmed cases was carried out to reveal monthly incidence trends, geographical and age distributions. Surveillance performance indicators, namely the non-measles febrile rash illness (NMFRI), and measles detection rate.

\subsection{Statistical Analysis}

Results were presented on frequency tables by year. The comparison of characteristics of subjects by year was carried out using Graph Pad Prime version 5.3 statistical package at $95 \%$ Confidence Interval (CI). The level of statistical significance was established at $\mathrm{p}<0.05$ using Fisher's exact 2-tailed values.

\subsection{Ethical Issues}

Appropriate informed consent and ethical approval were obtained from the subjects and authorities of the health institutions, respectively.

\section{Results}

Blood samples were obtained from 4159 client at the surveillance focal sites scattered across the 123 district (LGAs) and were tested for measles-specific immunoglobulin M (IgM). Of these, 1962 were males and 2197 were females. A total of $465(11.2 \%)$ tested positive for measles specific IgM antibodies.

Table 1 illustrates the demographic characteristics (age, sex, setting, vaccination status) of measles cases reported to the surveillance system in five years. Those under 5 years of age accounted for the largest proportion (56.4\%) of cases reported, investigated, and blood specimen drawn for estimation of measles specific IgM, while those aged 5-15 years constitutes $36.1 \%$ of those screened and $7.5 \%$ was recorded among those in age group 15 years and above. The highest number of IgM positive cases was found in those less than 5 years $(79.8 \%$ ), while those aged $5-15$ years and 15 years and above recorded $17.2 \%$ and $3.0 \%$ respectively. Statistically significant difference was established between the age groups examined $(\mathrm{p}<0.001)$. Females $(52.1 \%)$ were most susceptible to measles than males $(47.9 \%)$. However, no statistically significant difference was established between the sex groups considered $(\mathrm{p}=0.7571)$, and the odds ratio was high, 1.036 (95\% CI 0.854-1.257). The distribution of measles burden between urban and rural setting indicates that urban dwellers $53.8 \%$ were more susceptible to measles than rural dwellers $(46.2 \%)$, this relationship was established as statistically significant $(\mathrm{p}<0.0001)$ and odds ratio was also high 1.669 (95\% CI 1.375-2.025). A high significance of association between development of measles and vaccination status of subjects was also observed in this study ( $p<$ 0.0001 ), while odds ratio was also observed to be high 6.144 (95\% CI 4.977-7.511).

Figure 1 shows the number measles specific IgM positive cases and routine immunization (RI) coverage among children aged 0-11 months in the south south zone 20062010. RI coverage increased from $59 \%$ in 2006 to $79 \%$ in 2010 and the number of IgM positive cases also steadily increased from 37 cases in 2006 to 224 cases in 2010.

The World Health Organization defined the target of at least 2 non measles febrile rash illness (NMFRI) per 100 000 population as an indicator for monitoring the sensitivity of measles surveillance performance. This target was consistently achieved from 0.06 in 2006 to 3.55 in 2010 per 100000 population, except in 2006. Case detection rate also increased steadily from 0.24 in 2006 to 4.56 in 2010 (Figure 2).

Figure 3 illustrates the monthly distribution of measles specific IgM cases 2006-2010, high incidence of measles were observed in the months of October, November, December, January, February, March and April. 
Table 1. Demographic Characteristics of Measles Cases Reported to the Surveillance System.

\begin{tabular}{|c|c|c|c|}
\hline Risk Factors & No. Screened (\%) & No. Positive (\%) & P-value $95 \%$ CI) \\
\hline \multicolumn{4}{|l|}{ Age (years): } \\
\hline Under 5 & 2344 & $371(79.8)$ & \multirow{3}{*}{$P<0.0001$} \\
\hline $5-15$ & 1503 & $80(17.2)$ & \\
\hline $15+$ & 312 & $14(3.0)$ & \\
\hline \multicolumn{4}{|c|}{ Sex distribution: } \\
\hline Male & 1962 & $223(47.9)$ & $\mathrm{P}=0.7571$ \\
\hline Female & 2197 & $242(52.1)$ & $10.036(0.8540-1.257)$ \\
\hline Urban & 1767 & $250(53.8)$ & $P<0.001$ \\
\hline Rural & 2392 & $215(46.2)$ & $1.669(1.375-2.025)$ \\
\hline \multicolumn{4}{|c|}{ Vaccination status: } \\
\hline Zero dose & 1183 & $305(65.6)$ & $P<0.001$ \\
\hline Vaccinated & 2976 & $160(34.4)$ & $6.114(4.977-7.511)$ \\
\hline
\end{tabular}

$P$-value (Fisher exact) 2-tailed, OR = Odds Ratio at 95\% Confidence interval.

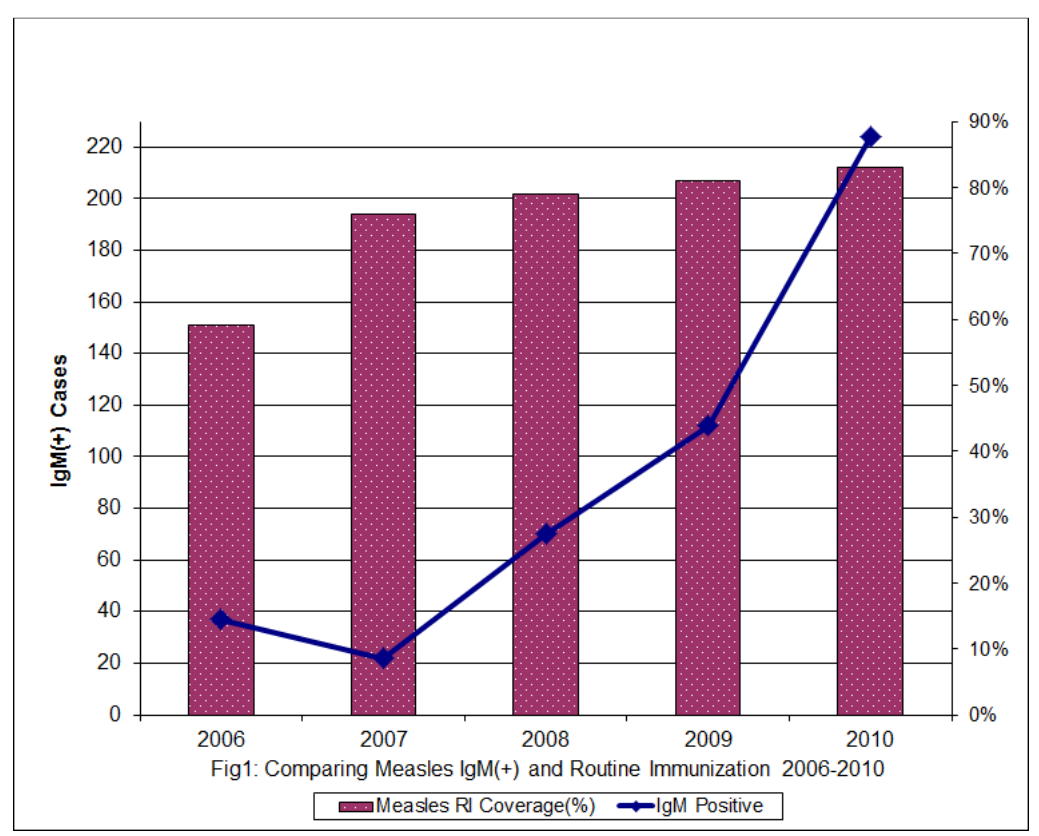

Fig. 1. Comparing Measles lgM(+) and Rountine lmmunization 2006-2010.

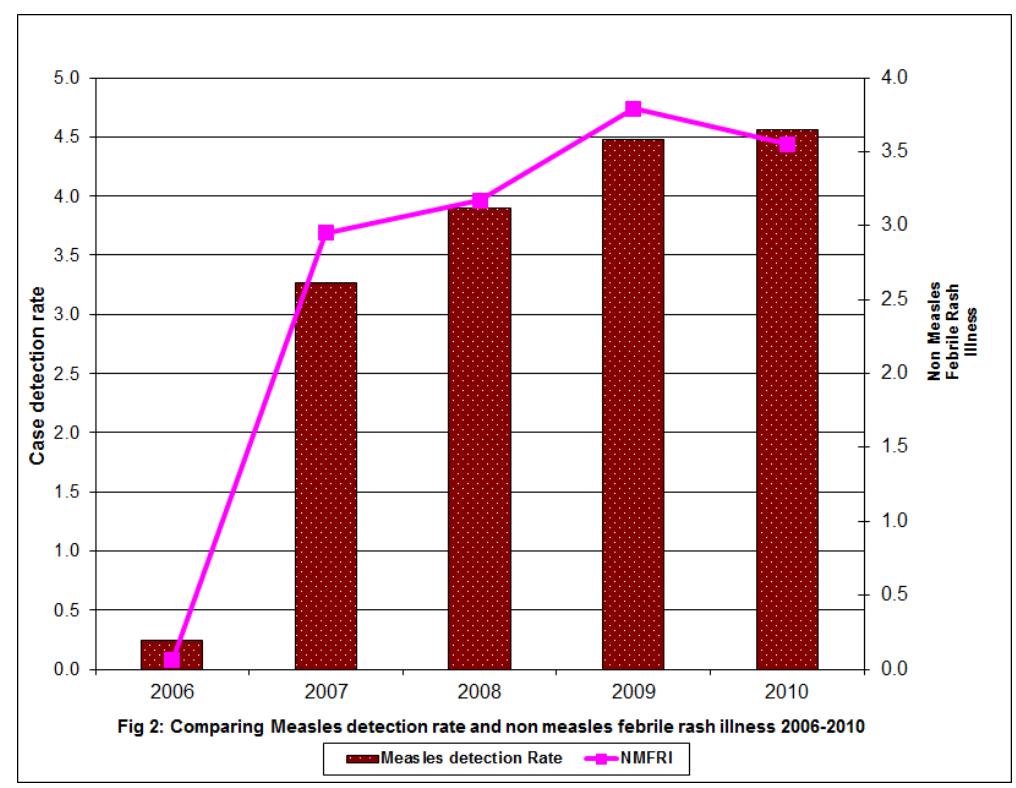

Fig. 2. Comparing Measles detection rate and non measles febrile rash illness 2006-2010. 


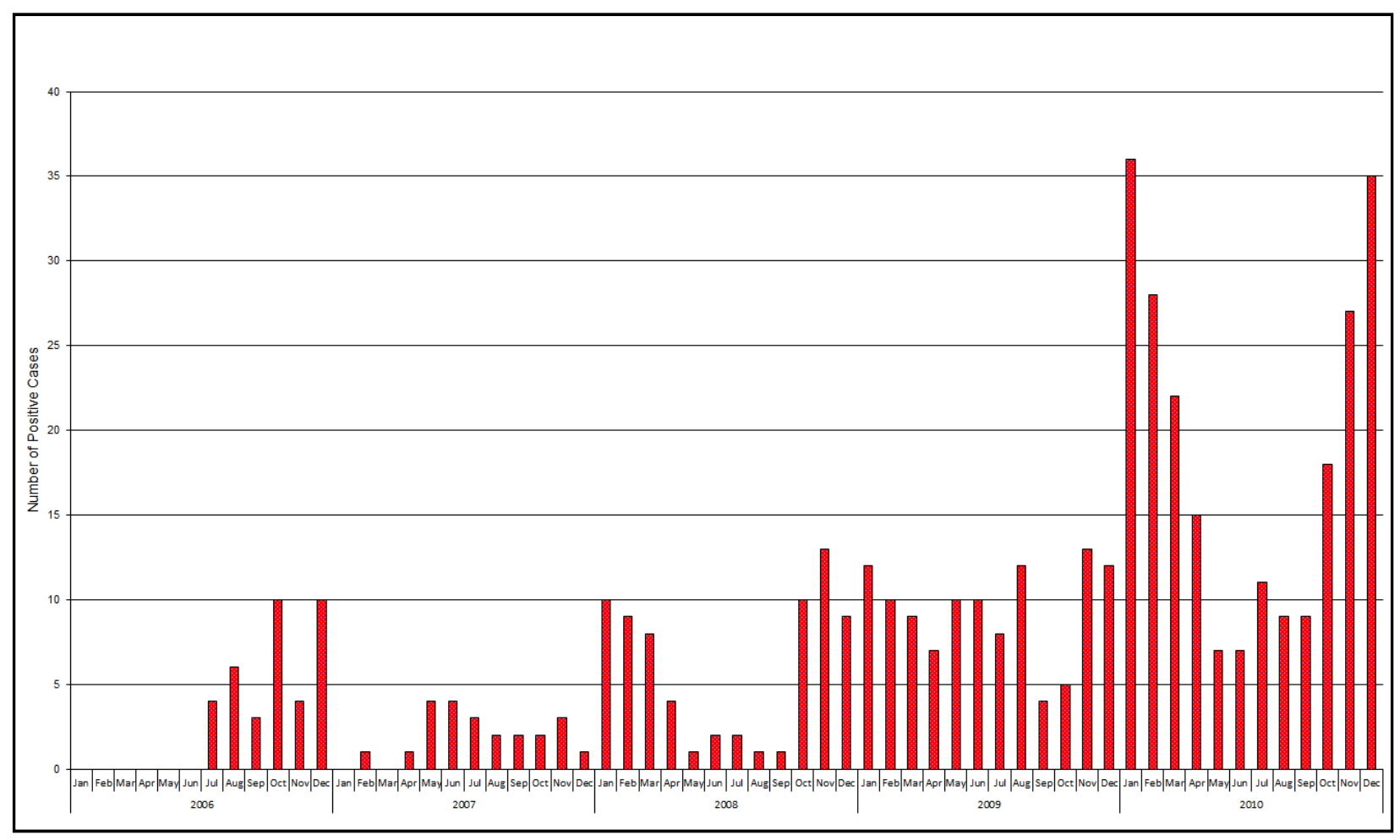

Fig. 3. Seasonal Variation in the distribution of Measles lgM Positive Cases.

\section{Discussion}

An interesting pattern of measles prevalence is revealed by the epidemiological data generated from the zone 2006-2010. In Nigeria, measles case based surveillance started in 2006 [2], following the measles vaccination catch-up campaign conducted nationwide in the 19 states in Northern Nigeria and 17 states in Southern Nigeria in December 2005 and October 2006 respectively. The reduced incidence of measles following these campaigns offered the country an opportunity to introduce a case-based surveillance system involving case investigation and specimen testing.

In this study, we evaluated three age categories; those aged 0-59 months, 5-14 years and 15 years and above. The $79.8 \%$ measles cases recorded in this study, in children $0-59$ months is higher than what is reported in other developing countries such as $32 \%$ in Ethiopia [5], $15 \%$ in Kenya [5], and $46.5 \%$ in southwest Nigeria [6]. The reason for the variation seen in data reported from different parts of Africa may be attributed to variation in measles surveillance sensitivity, or logistics challenges involved in reaching the population that is over $80 \%$ rural with vaccines and other interventions [7]. The high measles incidence among the under-five age group could be attributed to low literacy level, low socio-economic reasons, overcrowding, poor access to health-care facilities, missed opportunities during routine immunization programmes [8]. This study depicts that the most common age group affected during the period under observation in this study are $\leq 5$ and $\geq 5$ to $\geq 14$ respectively. To prevent future outbreaks, surveillance and routine immunization should be strengthened to achieve complete coverage as well as consideration of other age groups to avoid the accumulation of susceptible persons in the population. In addition, measles "catch-up" mass vaccination campaign should be conducted to interrupt chain of transmission and prevent future outbreaks. In Nigeria where measles is endemic, and the duration of impacts of campaign does not exceed two years, even in the face of high routine immunization and SIA coverage, effort should be tailored towards conducting 2-3years follow-up campaigns to reinforce the impact of mass campaigns.

The results of this study indicate that gender did not significantly influence the prevalence of measles among those screened, though slightly higher prevalence was observed in female $(52.1 \%)$ population than males $(47.9 \%)$, This experience is different from what was observed elsewhere in south-west, Nigeria by Adeoye et al, female $(41.4 \%)$, male $(58.6 \%)$ [8], our finding is in consonance with the report which documented that a measles antibody is marginally higher in females than in males. [9]. In Vietnam, the distribution in respect to gender, skewed towards males [10], similar observation was made by other scholars in Bangladesh [11].

The overall prevalence of measles in this study shows a significantly higher infection rate (65.6\%) among the unvaccinated populations compared to $34.4 \%$ observed among the vaccinated populations. Several studies have reported higher proportion of cases among unvaccinated individuals. In the Europe, case-base surveillance studies showed $40 \%$ in $2005,68 \%$ in 2006 and $80 \%$ in 2007 of cases were among unvaccinated population indicating immunity gaps [12]. In the United States of America, $89 \%$ was reported 
among unvaccinated individuals [13]. However, the $34.4 \%$ observed among vaccinated individuals in the study is very high considering the acceptable measles vaccine failure rate of $2-10 \%$ [9] and may be attributed to failure in seroconversion due to several factors such as vaccine failure, improper handling and poor cold chain maintenance, use of inappropriate route and dosage, none use of indigenous virus strains in the formulation and production of these vaccines and none adherence to appropriate age to be vaccinated, missed opportunities.

Findings in this study further reveal a higher prevalence of measles IgM antibody among those living in urban areas than in rural settings. This is consistent with previous study that reported high measles IgM prevalence among urban dwellers [14], and another which reported that measles is a more common health problem in urban dwellings [15] The variation in serologic profile between urban and rural settings might be due to the failing health care system, malnutrition, vaccine stock out, overcrowding and inadequate or logistics to support vaccine distribution to urban-rural communities, the children might also receive vaccine of low potency, due interruption in the cold chain system, failure in booster of natural measles in urban areas with population densities.[14].

In this paper, measles occurred throughout the year. However, the seasonal peak in incidence occurred from October to March throughout the 5 years studied. Such a seasonal trend has been reported in other studies, in Taiwan [16], Ethiopia, Kenya, and Benin (WHO). Other investigators in Nigeria $[17,18,19]$ made similar observations earlier. This seasonal variation in incidence of measles may be attributed to the dry season which enhances easy movement of infective droplets and transmission; the increased festivities promoted social interaction and consequently, spread of measles. This view was further reinforced by the sharp increase in incidence observed in this study from the month of January following the festivities in the month of December [20]. The implication is that health care providers should anticipate increase in number of cases of measles and prepare for their management during the dry season.

The catch up and follow-up campaigns conducted in 2006 and 2008 respectively targeted this period of high disease transmission to effectively reduced case incidence. Following the October 2006 catch-up campaign in the south, measles incidence was significantly reduced during and after the campaign, while the usual seasonal surge was noted but at reduced rate in 2009 and very significant in 2010.

We observed gradual and steady increase in routine immunization coverage from $57 \%$ in 2006 to $83 \%$ in 2010 , this apparent high routine immunization coverage recorded in this study did not translate to high population immunity as expected. We also observe a corresponding increase in the number measles specific IgM $(+)$ case within the same period. This observed immunity gaps may be attributed to the use of inappropriate population denominator, poor immunization coverage in pockets of communities, districts, since the coverage presented is a cumulative coverage that is not a uniform representative of component states/districts/communities covered in this report.

The present study confirms the improvement in measles surveillance in South South Nigeria. According to data for 2006-2010 from the same sources, the average rates of nonmeasles febrile rash illness (NMFRI) rose from 0.6 to 3.5 per 100000 population, with all cases undergoing laboratory diagnosis. The present results demonstrate that a significant proportion of measles cases are reported in the areas. Subsequently, it was confirmed that reporting and laboratory diagnosis improved markedly, reaching approximately $80 \%$ for both indicators. Since active surveillance was conducted in all prefectures of the one hundred and twenty three districts, the observed changes were not independent of the recent improvement in measles surveillance throughout the districts. Another factor that strongly influences the quality of measles surveillance is case detection rate. The WHO Measles surveillance performance indicator is 1 non-measles febrile rash illness per 100,000 population, which is an international standard used to assess the sensitivity of a national measles surveillance programme. Non-measles febrile rash illness has a direct relationship with population, increase in population figure results in high NMFRI rate expected to be recorded to meet the minimum target of $\mathrm{NMFRI} \geq 1$ per 100,000 population.

Vaccination of susceptible individuals through the full implementation of the strategy recommended by WHO in all countries remains the foundation of the measles elimination initiative [21]. Sensitive measles surveillance and high population immunity must be maintained to prevent the resumption of endemic transmission. Laboratory surveillance remains a central activity within the elimination programme. Standardized approaches to laboratory testing and interpretation of results are critical to ensure the continued success of the programme.

\section{Conclusions}

Concrete measures are to be taken in order to improve the current situation and to make progress towards elimination targets include: sustaining routine immunisation services, providing supplementary immunisation activities for susceptible population subgroups, strengthening surveillance by rigorous case investigations and laboratory confirmation, and improving the availability of high-quality information for both health professionals and the general public on the benefits and risks associated with immunisation.

\section{Acknowledgments}

We thank all Disease Surveillance and Notification Officers on the field especially in 2006-2010 in the 31 districts (LGAs) in Akwa Ibom State of Nigeria where these samples were collected for their cooperation and support

\section{Authors' Contributions}

Bassey conceived of the study, participated in its design, 
performed analysis and interpretation of data and drafted the manuscript. Alex, Weldegebriel, Igbu, Akpan, Ayodele, Maleghemi and Koko contributed in generation of raw data.

\section{References}

[1] A. Mohammed, P. Nguku, E.A. Abanida, K. Sabitu (2010). Evaluation of measles case-based surveillance system in Nigeria (2010). ecdc.europa.eu/en/ESCAIDE/Materials/Presentation/ESCAID E2010P. N

[2] Hitoshi Murakami; Nguyen Van Cuong; Hong Van Tuan; Katsuyuki Tsukamoto; Do Si Hien (2008).Epidemiological impact of a nationwide measles immunization campaign in Viet Nam: a critical review. Bull World Health Organ 86 (12). doi: 10.1590/S0042-96862008001200012

[3] Centers for Disease Control and Prevention Morbidity and Mortality Weekly Report JAMA. 2010;303(3):216-224.

[4] Guris G. Module on best practices for measles surveillance. WHO; 2001. http://www.who.int/vaccinesdocuments/DocsPDF01/www617.

[5] World Health Organization (2007). AFRO Measles Surveillance Feedback Bulletin, June 2007

[6] OO Opaleye, MO Adewumi, E Donbraye, AS Bakarey, GN Odaibo, OD Olaleye Prevalence of measles neutralizing antibody in children under 15 years in Southwestern Nigeria, Afr. J. Clin. Exper. Microbiol. 6(1) 2005: 60-63

[7] Samuel Ofosu-Amaah (1983). The control of Measles in Tropical Africa: A Review of Past and Present Efforts. Rev Inf. Dis 5 (3): $546-553$

[8] Adeoye IA, Dairo MD, Adekunle LV, Adedokun HO, Makanjuola J. Investigation of a measles outbreak in a Rural Nigerian community - The Aladura experience African Journal of Microbiology Research 2010;. 4(5): 360-366,

[9] Bassey EB, Moses AE, Udo SM, Umo AN. The Impact of Immunization Control Activities on Measles Outbreaks in Akwa Ibom State, South-South, Nigeria. Online J Health Allied Scs. 2010; 9(1):3

[10] Nmor JC, Thanh HT, Goto K. Recurring Measles Epidemic in Vietnam 2005-2009: Implication for Strengthened Control Strategies. Int J Biol Sci. 2011; 7(2): 138-146.
[11] Akramuzzaman SM, Felicity T. Cutts FT, Md J. Hossain MJ, Obaidullah K. Wahedi OK, Nahar N, Islam D, Shaha NC Mahalanabis D. Measles vaccine effectiveness and risk factors for measles in Dhaka, Bangladesh. Bulletin of the World Health Organization 2002;80:776-782

[12] Rogalska J, Santibanez S, Mankertz A, Makowka A, Szenborn L, Stefanoff P. Spotlight on measles 2010: An epidemiological overview of measles outbreaks in Poland in relation to the measles elimination goal. Euro Surveill. 2010;15(17)

[13] CDC. Measles United States, January--May 2011. MMWR 2011; 60(20); 666-668 .

[14] Eghafona NO. Measles antibody levels in children of rural and urban areas of Nigeria following vaccination campaign. Epidemiology and infection. 1987;99:85-9.

[15] Tayil SE, Shazly MK, El-Amrawy SM et al. Seroepidemilogical study of measles after 15 years of compulsory vaccination in Alexandria, Egypt. Eastern Medit Health Journal. 1998;4(3):437-447

[16] Chen CJ, Lin TM, Yeh YL. Analysis of the secular trend and seasonal variation of measles mortality rate in Taiwan. Annals of the Academy of Medicine, Singapore, 1984, 13:136-141.

[17] Ojuawo A, Bello M (2000). Measles in Ilorin. Nig. J. Med., 9:101- 103 .

[18] Ogunmekan BA, Braoven P, Marshell MC (1981). A seroepidemiological study of measles infection in normal and handicapped persons in Lagos, Nigeria. J. Trop. Med. Hyg., 84: $175-198$.

[19] Akande TM (2007). A review of measles vaccine failure in developing countries. Nig. Med. Pract., 52: $112-116$.

[20] Alphonsus N. Onyiriuka (2011). Clinical profile of children presenting with measles in a Nigerian secondary health-care institution. Journal of Infectious Diseases and Immunity Vol. 3(6), pp. 112-116, June, 2011

[21] de Quadros CA, Izurieta H, Carrasco P, Brana M, Tambini G. Progress toward measles eradication in the Region of the Americas. Journal of Infectious Diseases 2003;187 Suppl 1:S102-10.

[22] Akramuzzaman, S. M.; Felicity T. Cutts, et al., (2002). Measles vaccine effectiveness and risk factors for measles in Dhaka, Bangladesh. Bull World Health Organ vol 80 no. 10 